\title{
Competing Populations in Flows with Chaotic Mixing
}

\author{
István Scheuring ${ }^{\ddagger}$, György Károlyi ${ }^{\star}$, Zoltán Toroczkai $^{\Uparrow}$, Tamás Tél ${ }^{\S}$, and Áron Péntek ${ }^{\dagger}$ \\ ${ }^{\ddagger}$ Department of Plant Taxonomy and Ecology, Research Group of Ecology and Theoretical Biology, Eötvös University, \\ Ludovika tér 2, H-1083 Budapest, Hungary \\ * Department of Structural Mechanics, Budapest University of Technology and Economics, Müegyetem rkp. 3, H-1521 \\ Budapest, Hungary \\ ฯ Theoretical Division and Center for Nonlinear Studies, Los Alamos National Laboratory, Los Alamos, New Mexico 87545, \\ $U S A$ \\ $\S$ Institute for Theoretical Physics, Eötvös University, P. O. Box 32, H-1518 Budapest, Hungary \\ ${ }^{\dagger}$ Marine Physical Laboratory, Scripps Institution of Oceanography, University of California at San Diego, La Jolla, CA
} 92093-0238, USA

(November 13, 2018)

\begin{abstract}
We investigate the effects of spatial heterogeneity on the coexistence of competing species in the case when the heterogeneity is dynamically generated by environmental flows with chaotic mixing properties. We show that one of the effects of chaotic advection on the passively advected species (such as phytoplankton, or self-replicating macromolecules) is the possibility of coexistence of more species than that limited by the number of niches they occupy. We derive a novel set of dynamical equations for competing populations.
\end{abstract}

\section{INTRODUCTION}

One of the classical problems of ecology is the identification of the mechanisms responsible for the coexistence of competing species. It is an observational fact that in Nature numerous species are able to coexist, all competing for a limited number of resources. This observed coexistence is at odds with many classical theories and empirical studies predicting competitive exclusion of all but the most perfectly adapted species for each limiting factor (Gause \& Witt, 1935; Hardin, 1960). However, one of the key ingredients in these classical studies was the assumption of a homogeneous, well mixed and nonstructured environment which leads to an equilibrium state in the system. Thus, if coexistence is to persist over longer time periods, it must have a nonequilibrium character.

The coexistence problem is best illustrated in the case of phytoplankton communities as was originally presented by Hutchinson (1961). Here a number of species coexist in a relatively isotropic or unstructured environment, all competing for the same sorts of materials, and the number of species exceeds considerably the number of limiting factors. To solve this so called "paradox of plankton", Hutchinson put forward the idea that seasonal environmental changes prevent competitive exclusion in natural phytoplankton communities. Thus the species of the community, at least on the time scale of ecological observation, are in nonequilibrium coexistence.

Since then numerous investigations revealed many different mechanisms, including spatial and temporal heterogeneity of habitat, predation, disturbance, coevolution, etc. (Wilson, 1990) Chesson, 2000), increasing the probability of competitive coexistence. Naturally, under the word "competition" many different biological phenomena are collected together, which influence the coex- istence of species in different ways.

Thus the original problem changed into finding the most relevant mechanisms which maintain diversity in particular situations (Connell, 1978; Huston, 1979; Wilson, 1990; Tilman \& Pacala, 1993; Bartha et al., 1997). Despite the vivid debate in this field of ecology, there is by now a consensus that climatic periodicities and fluctuations play the main role in causing species' persistence in phytoplankton communities Gaedeke \& Sommer, 1986; Reynolds, 1993; Sommer et al., 1993). It is frequently argued that an intermediate disturbance (Connell, 1978) is the most adequate hypothesis for the explanation of high diversity in aquatic systems (cf. Reynolds, 1998).

One can meet a similar problem in early evolution of life. Since life evolves from the simple structured entities to the most complex ones, there must have been a stage in the evolution, when life was essentially no more complex than what a collection of self-replicating nucleic acids present (Maynard Smith \& Szathmáry , 1995). They were competing for a few limiting resources (such as mononucleids and energy rich chemicals) and making copies of themselves without any specific enzyme. Without enzymes the copying accuracy could not be very high. Estimating the selective superiority of the best replicator and the copying accuracy per nucleotide, it is concluded that the maximum length of these molecules is about 100 nucleotides (Eigen, 1971). However, in a well mixed homogeneous environment, as the prebiotic ocean is supposed to have been, there are only a few winners of the selection, namely the most fit macromolecule surrounded by its closest mutants (Eigen, 1971; Eigen \& Schuster, 1979). But how can we surmount the gap between these primitive replicators with 100 nucleotides and the most simple RNA viruses with 4000-5000 nucleotides? Specific replicase enzymes are needed to increase the copying fidelity, and thus the length of the replicator, but these 
replicators are too short to code specific enzymes. This is the "Catch 22" of the prebiotic evolution (Maynard Smith, 1983): no genome without an enzyme, however no enzyme without genomes. This problem can be resolved if some mechanism maintains the coexistence of several different replicator molecules and therefore the information necessary for coding a replicase enzyme can be stored by the union of smaller information carriers. In this situation the replication error does not grow exponentially as in the case of a base-by-base copying, it grows only linearly with the number of smaller carriers.

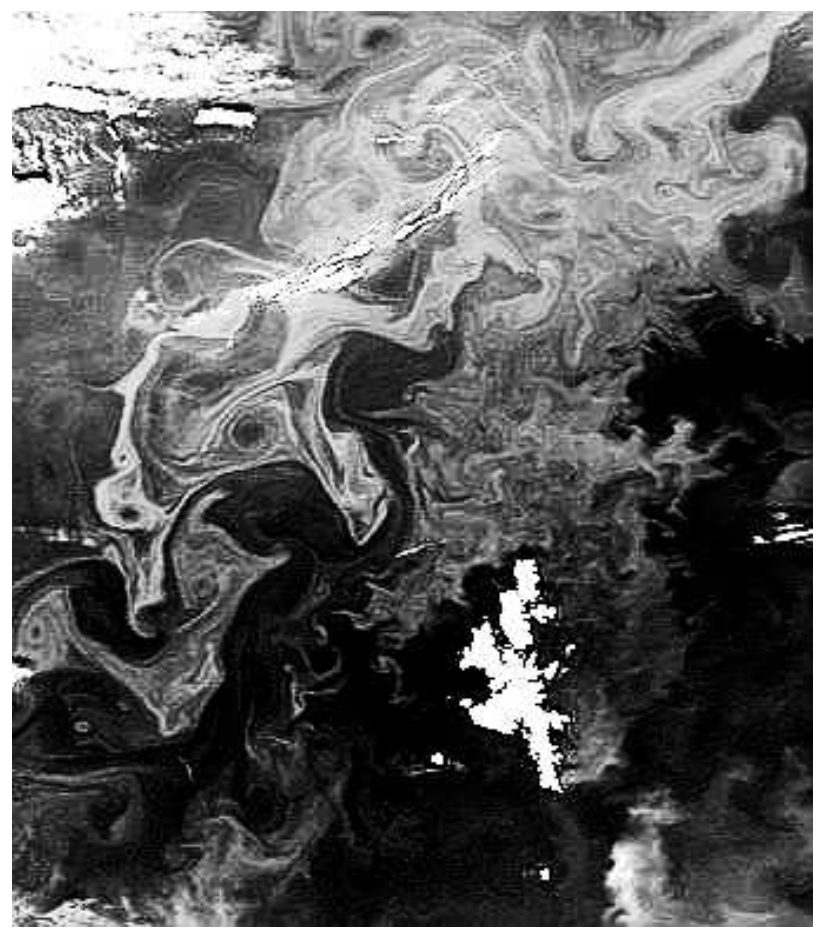

FIG. 1. SEAWIFS image of a phytoplankton bloom at Shetland Islands, May 12, 2000, from the NASA archive. Plankton individuals (light grey) move along a fractal set.

Current theories point out coexistence of replicators moving on a surface (Boerlijst \& Hogeweg, 1991; Czárán \& S athmáry, 2000), preferring thus the concept of "prebiotic pizza" against the concept of "prebiotic soup" (Wächtershäuser, 1994). However some kind of cooperation among the replicator molecules are built into these models, consequently they are not completely competitive. An alternative explanation assumes that both the replicative and enzymatic functions were co-evolved, thus the lenght of the replicators and the accuracy of enzymatic functions increased together (Poole et al., 1999. Scheuring, 2000). In both problems (i.e., in the paradox of plankton and in the Catch 22 of prebiotic evolution) the traditional population dynamical equation for two spedies $B_{1}, B_{2}$ competing for the resource $A$ read:

$$
\begin{aligned}
& \frac{d N_{1}}{d t}=\alpha_{1} N_{1}-\delta_{1} N_{1}, \\
& \frac{d N_{2}}{d t}=\alpha_{2} N_{2}-\delta_{2} N_{2} .
\end{aligned}
$$

Here $N_{i}$ is the instantaneous number of individuals of species $B_{i}$ in a given range of a well strirred region. The instantaneous parameters $\alpha_{i}, \delta_{i}$ are positive and depend, in general, on the concentration of the resource material $A$, too.
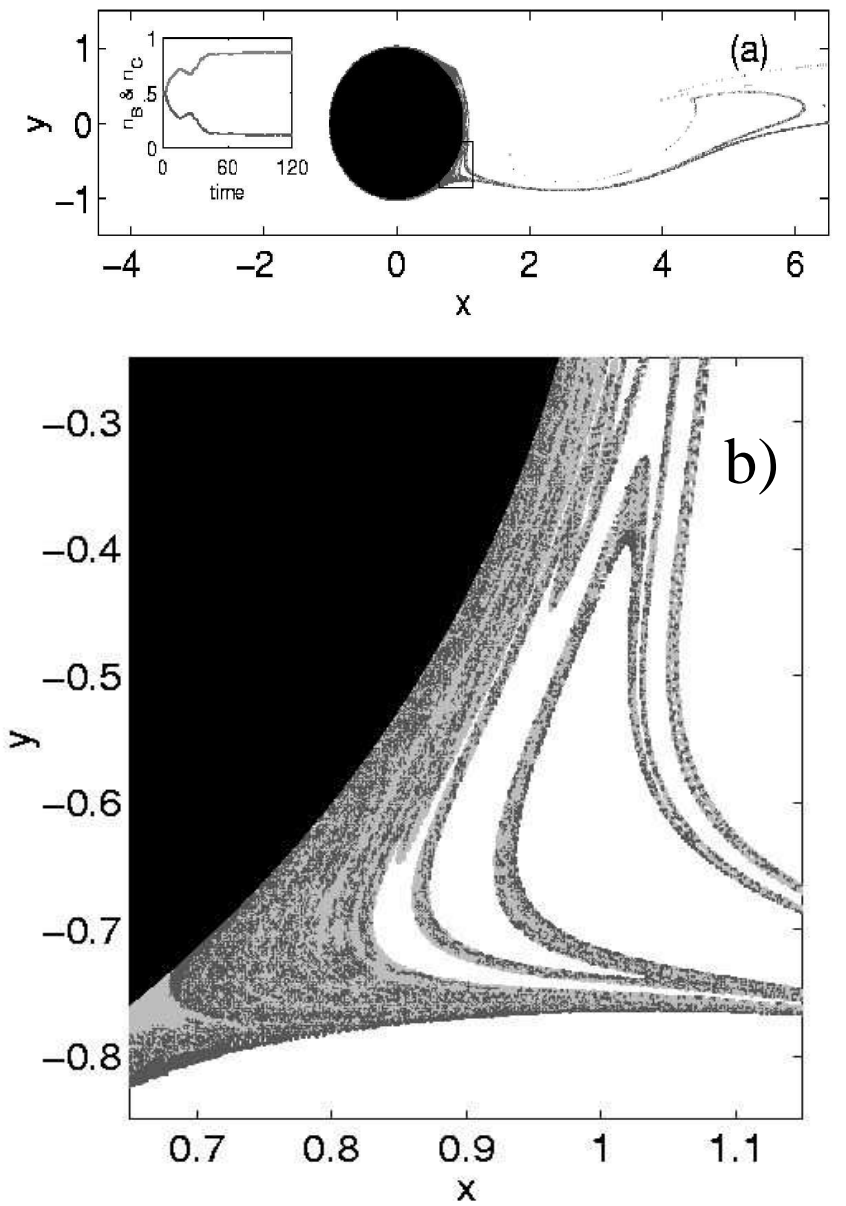

FIG. 2. The distribution of two populations (light gray $(B)$ and dark gray $(C)$ ) competing for the same resource material (white $(A)$ ) in the wake of a cylinder. The flow is from left to right. The inset in (a) shows the time-dependence of the population numbers $n_{B}, n_{C}$ and clearly indicates the approach to a steady state of coexistence after about 40 time units which is the period of the flow. A blowup of the region indicated by a rectangle in (a) is seen in (b). Species distribution is strikingly similar to many of the patterns found on the NASA SEAWIFS satelite pictures of plankton blooms (see Fig 1). After Károlyi et al. (2000.

Independently of the particular form of this dependence and the dynamical equation of $A$, no fixed points can exist in the system in which both species would be in a steady state with nonzero values of $N_{i}=N_{i}^{*}$ Gurney \& Nisbet, 1998). The coexistence might, however, be 
possible in imperfectly stirred environments. As numerous remote sensing images demonstrate phytoplankton are distributed along fractal filaments in the oceans indicating strong but imperfect mixing environment (Fig 1).

Recent development in the field of hydrodynamics encouraged us to revisit the population dynamics of competing species in open aquatic systems. In aquatic systems of large extension, on the time scales characteristic to the life cycle of microorganisms and replicators, the hydrodynamical flows are locally open, i.e. there is a net current, transporting both competitors and nutrients, flowing through the typical observation region. It is even more obvious that the flow is open in the wake of islands surrounded by strong ocean currents Arístegui et a (., 1997) and around the deep see hot springs where the cradle of life probably swung (Holm, 1992).

With the aid of numerical simulations we have previously shown that the coexistence of passively advected competing species is typical in open chaotic flows (Károlyi et al., 2000; Scheuring et al., 2000). For simplicity, we have considered the two-dimensional flow around a cylindrical obstacle placed into a uniform background flow. For moderate inflow velocities there is a periodic detachment of vortices in the wake of the obstacle with period $T$, which forms the von Kármán vortex street (Shariff et al., 1991; Jung \& Ziemniak, 1992; Sommerer et al., 1996). The flow in the wake is time-dependent but still spatially regular. Here individuals of two passively advected species compete for a common limiting resource, see Fig. 2. We argued that coexistence is due to the fractal structures typically appearing in the advection patterns of such flows, however, we have given only a heuristic interpretation for the mechanisms maintaining coexistence in this hydrodynamical system. In this article we present a mathematical deduction to explain coexistence of competitors in open chaotic flows. The mathematical problem is to investigate two coupled population dynamical processes evolving on a fractal support. We shall present a new class of equations which describe this situation, and allow for the coexistence of at least two species competing for the same resource. A novel feature of these equations will be a singularly nonlinear (power-law) form of both the replication term and the coupling between the populations.

In the following section we summarize the qualitative features of the relevant physical process, followed by a study of the dynamics of a single population in an open chaotic flow. Consequently, the coexistence of competitors is discussed by first giving a qualitative argument based on the single population picture, followed by a detailed mathematical model leading to the aforementioned new type of population dynamical equations. Next, this theory is compared with further numerical results carried out on a simple map modelling the advection dynamics, on the so-called baker map. We conclude with a summary and outlook.

\section{PASSIVE ADVECTION IN OPEN FLOWS}

Chaotic advection in open hydrodynamical flows is an ubiquitous phenomenon. A flow is considered locally open if there is a net current flowing through the observation region (Lamb, 1932). It became clear in the last decade that passive advection even in simple timedependent flows is typically chaotic (Péntek et al., 1996; Sommerer et al., 1996; Károlyi \& Tél, 1997) and possesses complicated particle trajectories. These flows, characterized by strong imperfect mixing, lead to a fractal spatial distribution of advected particles in a finite region of the flow. This region is called the mixing region. In our terminology, a flow is chaotic if the advection dynamics generated by the flow is chaotic.

In the case of several (three or more) types of passively advected tracers, distinguished for example by their color, it was shown (Toroczkai et al., 1997, and references therein) that their distribution may follow a rather non-trivial topology on the fractal, a property called the Wada property: every point on the fractal is lying on the boundary of at least three colors.

It is worth emphasizing that a complicated flow field (turbulence) inside the mixing region is not required for the flow to be chaotic, (i.e., for complex advection dynamics or for the appearance of fractal patterns). Even simple forms of time dependence, e.g. a periodic repetition of the velocity field with some period $T$, is sufficient (Aref, 1994). Thus, for sake of simplicity, we examine advection in time-periodic open flows.

The complicated form of trajectories implies a long time spent in the mixing region. In other words, advected particles can be temporarily trapped there. It is even more surprising, however, that there is an infinity of special nonescaping orbits. The simplest among these orbits are the periodic ones with periods that are integer multiples of the flow's period $T$. All the nonescaping orbits are highly unstable, of saddle type, and possess a strictly positive local Lyapunov exponent (which is the expanding eigenvalue of the unstable periodic orbit). Another important feature of these orbits is that despite their infinite number they are rather exceptional so that they cannot fill a finite portion of the phase space. Indeed, the union of all nonescaping orbits forms a fractal "cloud" of points on any snapshot. This fractal cloud moves periodically with the flow and never leaves the mixing region.

Typical advected particle trajectories are not in the set of the nonescaping orbits, but are, nevertheless, influenced by them. They follow closely some periodic orbit for a while and later turn to follow others. This wandering amongst periodic (or, more generally, nonescaping) orbits results in the chaotic motion of passively advected particles. Indeed, as long as the particles are in the mixing region, their trajectories possess a positive average Lyapunov exponent $\lambda$. Hence the union of all nonescap- 
ing orbits is called the chaotic saddle. The flows relevant from our point of view can be conisdered to be incompressible. This results in a time-reversal invariant, area preserving particle dynamics. Therefore, the negative average Lyapunov exponent is exactly $-\lambda$, and it characterizes the compression towards the chaotic saddle.

While many of the particles spend a long time in the mixing region, the overwhelming majority of them leaves this region sooner or later. The decay of their number in a fixed frame is typically exponential with a positive exponent $\kappa(<\lambda)$, which is independent of the frame, i.e., $N(t)=N(0) \exp (-\kappa t)$. This quantity $\kappa$ is the escape rate from the saddle (or from the mixing region). The reciprocal of the escape rate can be considered as the average lifetime of chaos, and therefore the chaotic advection of passive particles in open flows is a kind of transient chaos (Tél, 1990).

The chaotic saddle is the set of nonescaping orbits which advected particles may follow for an arbitrarily long time. Each orbit of the set, and therefore the set as a whole, has an inflow and an outflow curve, also called in the mathematical jargon of chaos theory the stable and unstable manifolds, respectively. The inflow curve is a set of points along which the saddle can be reached after an infinitely long time. The outflow curve is the set along which particles lying infinitesimally close to the saddle will eventually leave it in the course of time. By looking at different snapshots of these curves we can observe that they move periodically with the period $T$ of the flow. Their fractal dimension $D_{0}\left(1<D_{0}<2\right.$ in two-dimensional flows) is, however, independent of the snapshot. (The inflow and outflow curves have identical fractal dimension due to the advection dynamics' time reversal invariance.)

There is a unique relation between the fractal geometry and the advection dynamics, expressed by the relation ${ }^{1}$ (Kantz \& Grassberger, 1985; Hsu et al., 1988; Tél, 1990):

$$
D_{0}=2-\frac{\kappa}{\lambda}
$$

It says that the deviation of the dimension from that of the plane is given by the ratio of two quantities characterizing the global and the local instability of the dynamics. Relation (3) shows that out of the three basic characteristics $\left(\kappa, \lambda\right.$ and $\left.D_{0}\right)$ only two are independent. When speaking about population numbers in what follows, we

\footnotetext{
${ }^{1}$ By characterizing the dynamics by one single dimension $D_{0}$, we have assumed that the advection process has a monofractal geometry. In reality, a set of dimensions $D_{q}$ is required for the full description of the fractal aspects. It is for the $q=1$ dimension, the so-called information dimension, $D_{1}$, for which (3) is an exact equality. In practice, however, the relative difference between $D_{0}$ and $D_{1}$ is on the order of a few percents and therefore the use of a single dimension is justified for practical purposes.
}

shall use the escape rate and the fractal dimension as independent parameters. In the dynamics of the stripe widths (see next section), however, only the average Lyapunov exponent appears.

The outflow curve plays a special role since it is the only set which can be directly observed in an experiment. Let us consider a droplet (ensemble) of a large number of particles which initially overlaps with the inflow curve. As the droplet is advected into the mixing region its shape is strongly deformed, but the ensemble comes closer and closer to the chaotic saddle as time goes on. Since, however, only a small portion of particles can fall very close to the inflow curve, the majority does not reach the saddle and starts flowing away from it along its outflow corve. Therefore, in open flows droplets of particles trace out the outflow curve of the chaotic saddle after a sufficiently long time of observation (in fact, the populations in Fig. 22 are distributed along the outflow curve of the chaotic saddle present in the wake).

\section{DYNAMICS OF A SINGLE POPULATION}

In this section the mathematical derivation of the dynamics of a single population living in an open chaotic flow is briefly repeated (Toroczkai et al., 1998; Károlyi et al., 1999; : Tél et al., 2000, for more details see). Replication, competition for the limiting resources, and spontaneous decay are taken into account in our population model, while stage and age structure is neglected for simplicity. We derive discrete and continuous-time models as well.

First we assume that the intake of resource, multiplication and decomposition are instantaneous and take place at integer multiples of a time lag $\tau$. Here $\tau$ acts as an average time-scale on which the reproduction takes place.

The basic observation is that after a sufficiently long time, the filaments of the outflow curve are covered in narrow stripes by individuals of species $B$ due to their replication (Toroczkai et al., 1998; Károlyi et al., 1999). Individuals are thus distributed on a fattened-up fractal set. On linear scales larger than an average width $\varepsilon^{*}$ the distribution of $B$ is a fractal of the same dimension $D_{0}$ as the outflow curve of the chaotic saddle in the flow with passive advection without biological activity. Let $\varepsilon^{(n)}$ denote the average width of these stripes 
right before replication and decomposition takes place. It is worth measuring this width in the unit of a characteristic length scale of the flow (e.g. in the cylinder radius in the example of Fig. 2). Thus, $\varepsilon^{(n)}$ is a dimensionless variable. Since material $A$ is available outside of these stripes, replication increases the width with some constant distance $\gamma$, the replication range, while spontaneous decay due to death of individuals decreases it with a distance $\mu$. The net effect of the replication and spontaneous decay is then a broadening of the width by an amount proportional to the difference $\sigma=\gamma-\mu$, the effective replication range. Thus, $\varepsilon^{(n)} \rightarrow \varepsilon^{(n)}+c \sigma$. Here $c$ is a dimensionless number expressing geometrical effects. If the fattened-up filaments do not overlap, then replication does occur on the both side of stripes and $c=2$. If there is overlapping among some of the fattened-up filaments, such as in the case of a fractal, then $c \neq 2$. This geometrical factor turns out to be slightly time dependent due to the pulsation of the flow, but for simplicity it can be considered to be constant from the point of view of the qualitative behaviour of the population (Toroczkai et $a(., 2001)$.

In the next period of length $\tau$ there is no replication and decomposition, just contraction towards the outflow curve. The average contraction factor is $\exp (-\lambda \tau)$, where $(-\lambda)$ is the negative average Lyapunov exponent of the advection dynamics. Therefore, the width $\varepsilon^{(n+1)}$ right before the next replication can be given as

$$
\varepsilon^{(n+1)}=\left(\varepsilon^{(n)}+c \sigma\right) e^{-\lambda \tau} .
$$

This is a recursive map for the actual width of the $B$ stripes on snapshots taken with multiples of the time lag $\tau$. The solution of (4i) converges for $n \rightarrow \infty$ to the fixed point

$$
\varepsilon^{*}=\frac{c \sigma}{e^{\lambda \tau}-1} .
$$

In the time-continuous limit $\tau \rightarrow 0, \sigma \rightarrow 0$, but keeping $\sigma / \tau \equiv v_{r}$ constant, one obtains the differential equation:

$$
\frac{d \varepsilon}{d t}=c v_{r}-\lambda \varepsilon
$$

which has a steady-state solution given by:

$$
\varepsilon^{*}=\frac{c v_{r}}{\lambda} .
$$

Here $v_{r}$ can be interpreted as the net speed of replication.
Knowing the $\varepsilon$-dynamics and that the individuals accumulate on a fractal set in the mixing region, the time evolution of the number $N$ of $B$ individuals in that region can be calculated. First, note that the area $\mathcal{A}$ occupied by species $B$ scales as $\mathcal{A} \approx \epsilon^{2-D_{0}}$ with $D_{0}$ as the fractal dimension of the outflow curve for any box size $\epsilon$ not smaller than the width $\varepsilon$ of the $B$-stripes. We can thus choose $^{2}$

$$
\epsilon=\varepsilon \approx \mathcal{A}^{1 /\left(2-D_{0}\right)} .
$$

If the linear size of the area occupied by a single individual is $\epsilon_{0}$, we have $N=\epsilon_{0}^{-2} \mathcal{A}$, and therefore we can rewrite (4) or (6) so that it represents an equation for the individuals in discrete and continuous cases, respectively:

$$
N^{(n+1)}=e^{-\kappa \tau}\left\{\left[N^{(n)}\right]^{1 /\left(2-D_{0}\right)}+q \sigma\right\}^{\left(2-D_{0}\right)},
$$

and

$$
\frac{d N}{d t}=-\kappa N+q\left(2-D_{0}\right) v_{r} N^{-\beta}
$$

with

$$
q=c \epsilon_{0}^{-2 /\left(2-D_{0}\right)} .
$$

Here (3) has been used, and

$$
\beta \equiv \frac{D_{0}-1}{2-D_{0}}
$$

appears as a nontrivial exponent. ${ }^{3}$ Since the fractal dimension of the outflow curve lies between 1 and 2, exponent $\beta$ is positive. For $D_{0}=1$ the differential equation (10) describes a classical surface reaction along a line with front velocity $v_{r}$ in the presence of escape. For $1<D_{0}<2$ it represents a novel form of dynamical equations containing also an enhancing biological activity term with a negative power of the area occupied by $B$ due to the fractality of the outflow curve. The less $B$ individuals are present, the more effective the reproduction is, because the resolved perimeter is larger. Consequently, in a competitive situation the subordinate species has an advantage if it becomes rare compared to the dominant species. This balancing mechanism can make coexistence possible, as shown in the the next sections.

As one can see from Eqs. (9, 10), in both the discrete and continuum pictures a steady state is reached after

\footnotetext{
${ }^{2}$ In general, (8) also contains a proportionality constant, called the Hausdorff volume. Since this only rescales the constant $q$ in equation (9), for clarity we took the Hausdorff valume to be unity.

${ }^{3}$ For multifractal flows one can show (Tél et al., 2000) that exponent $\beta$ is that given by 12 with $D_{0}$ replaced by the information dimension $D_{1}$.
} 
a sufficiently long time if the geometrical factor $c$ (and therefore also $q$ ) is constant (Toroczkai et al., 2001). In this case, the steady-state number of individuals in the mixing region is $N^{*}=\epsilon_{0}^{-2}\left(\varepsilon^{*}\right)^{2-D_{0}}$ where $\varepsilon^{*}$ is given by (5) and (7) for the discrete and continuum cases, respectively.

\section{A MODEL OF COMPETITION}

As in the single species case, we consider a simple model of replication and competition with passively advected point like individuals of type $B_{1}$ and $B_{2}$, multiplying themselves instantaneously. The resource material $A$ which the different species $B_{1}$ and $B_{2}$ compete for is uniformly distributed on the surface of the flow. Similarly to the single species case, the parameters $\gamma_{i}$ and $\mu_{i}(i=1,2)$ are defined as the increase and decrease of the $B_{i}$ stripe width due to replication and decomposition, respectively. Therefore two autocatalytic processes $A+B_{1} \longrightarrow 2 B_{1}, B_{1} \longrightarrow A$ and $A+B_{2} \longrightarrow 2 B_{2}, B_{2} \longrightarrow A$ represent the replication and competition process in our model in an imperfectly mixed environment. Similarly to the single species case, the parameters $\gamma_{i}$ and $\mu_{i}(i=1,2)$ are defined as the increase and decrease of the $B_{i}$ stripe width due to replication and decomposition, respectively, so that the effective replication distances are $\sigma_{i}=\gamma_{i}-\mu_{i}$.

As before, an important feature of the advection dynamics is its deterministic nature. Concerning the population dynamics, this implies that we work in the limit of weak diffusion and assume that the mutual diffusion coefficients between any pair of the constituents is small.

Prior to discussing the consequences of the imperfect mixing generated by the chaotic flow to this dynamics, it is worth briefly giving the traditional equations governing the above defined autocatalytic processes in a well-mixed environment. In a fixed region of observation they are:

$$
\begin{aligned}
& \frac{d N_{1}}{d t}=\gamma_{1} A N_{1}-\mu_{1} N_{1}, \\
& \frac{d N_{2}}{d t}=\gamma_{2} A N_{2}-\mu_{2} N_{2},
\end{aligned}
$$

where $N_{i}$ denotes the number of individuals of species $B_{i}$, and $A$ is the instantaneous amount of the resource material in the same region. Note that the meaning of the replication and death rates are slightly different here from those in the discrete model (thus e.g., $\mu_{i}$ in eqs. (13), (14) is of dimension frequency, while the same quantity in the discrete version is a distance). If the dynamics of resource is much faster than the dynamics of competing species, then the former can be considered to be in a quasistationary state: $d A / d t=0$. The equation for resource $A$ is then

$$
\frac{d A}{d t}=0=l-\gamma_{1} A N_{1}-\gamma_{2} A N_{2},
$$

and $l$ is the constant inflow of resource $A$ into the region of observation. Equations (13), (14) correspond to the general scheme (2) and (2) given in the Introduction by identifying $\mu_{i}$ with $\delta_{i}$ and $\gamma_{i} A$ (where $A$ is given by the right hand side of (15)) with $\alpha_{i}$.

After analyzing (14) and (15), one can easily see that species with lower ratio $\gamma_{i} / \mu_{i}$ of replication and death rates would be outcompeted, and thus stable coexistence is impossible.

\section{COEXISTENCE OF COMPETING SPECIES}

In the following, using a gedankenexperiment, we find conditions under which the two species may coexist in the imperfectly mixing environment characterized by the existence of a chaotic saddle and its (fractal) outflow curve. First, let us assume that initially there is only one species, for e.g., $B_{2}$ in the mixing region. Also for the simplicity of the writing, we will refer to the continuum version of the single species dynamics, namely Eq. (10). After a time the number $N_{2}$ of $B_{2}$ will be close to a steady state value, $N_{2}^{*}$. We now let a small quantity of species $B_{1}$ invade the mixing region, so small that it cannot change the steady state of $B_{2}$. The question is: under what conditions this invasion will lead to a self-sustained $B_{1}$ population, coexisting with $B_{2}$ ?

When we are letting species $B_{1}$ invade the mixing region such that $B_{2}$ is already present, we must ensure for the coexistence that there is not only $B_{1}-B_{2}$ interface present but also $B_{1}-A$ interface, at all times. To show that this is indeed possible, we recall the Wada property of mixing on the chaotic saddle, mentioned previously. As shown by (Toroczkai et al., 1997), if species $B_{1}$ is inserted such that the $B_{1}-A$ interface intersects the inflow curve, then this boundary must be present arbitrarily close to all points on the outflow curve. Therefore this initial condition ensures the existence of the $B_{1}-A$ interface at all times on the saddle and its outflow curve. Thus, when there are interactions among the species, then, due to the relatively long time they spend on this fractal, and due to the largely increased interfaces a non-trivial and novel type of behavior can emerge.

Species $B_{1}$ becomes thus trapped by the chaotic saddle, and will be distributed in very narrow filaments along the surface of some of the $B_{2}$ stripes close to the $D_{0}$ dimensional outflow curve. Since by assumption, the population number $N_{1}$ of species $B_{1}$ is small, $N_{1} / N_{2} \ll 1$, there is no feedback on $B_{2}$ and this species remains in dynamical equilibrium: $d N_{2} / d t \approx 0$. The dynamics of species $B_{1}$ can thus be described by (10) written for $N_{1}$. The important nature of $(10)$ is that the first term on the right side, (which is responsible for the outflow from the fractal set) tends to zero for very small $N_{1}$. The second term, however, which describes an autocatalytic process can be arbitrarily large if $N_{1}$ is small, due to the negative exponent $-\beta$. Thus, if $N_{1}$ is sufficiently small then 
$d N_{1} / d t>0$. It means that the $B_{1}$ population always increases if it's number is close to zero. In other words, the fixed point $N_{1}^{*}=0$ is unstable.

Following the same argument, if $B_{1}$ and $B_{2}$ are already in dynamical equilibrium on the fractal set then a third species can invade this coalition in the same manner. This coexistence has indeed been demonstrated for three species by numerical simulations (Károlyi et al., 200().

In this gedankenexperiment we assumed that the invading population is so weak that it is distributed in narrow filaments along the "surface" of the already existing population and does not influence this population at all. After the number of the invading population has started to grow, there is an increasing interaction between the populations due to the competition for the same resource. As a consequence, the originally steady population is not in a stationary state and the dynamics should be treated in a selfconsistent manner. This is shown in the next section.

\section{A MATHEMATICAL MODEL FOR THE COMPETITION DYNAMICS}

After sufficiently long time, both species $B_{1}$ and $B_{2}$ will be distributed in narrow stripes along the chaotic saddle's outflow curve as follows from the passive advection dynamics. Due to the replication and decomposition, however, the stripes have finite widths (cf. Fig. 2) which might depend on time. Let $\varepsilon^{n}$ denote the dimensionless average width of the stripes right before an instantaneous replication takes place. These stripes are defined by the fact that outside of them there is only background material $A$ available. Inside the stripe of width $\varepsilon^{(n)}$ there might be several narrow $B_{1}$ or $B_{2}$ filaments. The background material $A$ is eaten up sooner or later in the inside of any stripe, therefore, for the sake of an easier presentation, we assume that this is the case and only material $B_{1}$ and $B_{2}$ are present. Let us denote the total widths of all the filaments of a given material within an $\varepsilon^{(n)}$ stripe by $\varepsilon_{i}^{(n)}$ with $i=1,2$ corresponding to $B_{1}$ and $B_{2}$, respectively. The sum of these partial widths is of course the total one $\varepsilon_{1}^{(n)}+\varepsilon_{2}^{(n)}=\varepsilon^{(n)}$. Our aim is to build up the dynamics of the partial widths based on plausible assumptions, from which the dynamics of the different populations follows.

We assume, that the boundaries are occupied by species $B_{1}$ or $B_{2}$ with probabilities $p_{1}$ and $p_{2}$, respectively. In other words, a stripe-boundary picked at random from the many filaments of the outflow curve will have a probability $p_{i}$ to be of type $B_{i}, i=1,2$. If mixing of the two species were perfect along the fractal set, these probabilities would be equal to their relative densities. This is not the case, however. The relative position of the species in the initial distribution to the inflow curve determines which individual or patch of individuals will be trapped by which orbit of the chaotic saddle. The rest, i.e., the untrapped individuals will drift out of the mixing region. The trapped individuals, however, will stay there forever, and follow their specific trapped orbit. In the course of time, individuals give birth to others of the same species, and patches of individuals are stretched along the outflow curve specific to the trapping orbit of the chaotic saddle. In either cases, we end up with long stripes of the two species lined up along each other in an alternating manner, tracing out the outflow curve. Then the probability of one species to be on the edge of these lines, and thus to be capable of reproduction, depends on which trapping orbit will produce the filament of outflow curve being on the edge of the stripe, on the order in which the species are lined up across one stripe, and on the actual width of the coverage of the filaments. In other words, it is the complex chaotic dynamics which makes the introduction of probabilistic concepts - on a somewhat phenomenological level - unavoidable.

The probabilities $p_{i}$ depend on what the distribution of the species inside the stripes is. Thus, the simplest possible assumption is that the probabilities depend on the partial widths $\varepsilon_{i}^{(n)}$. Their actual functional form might also contain parameters of the flow and of the biological activity.

Naturally the probabilities fulfill $0 \leq p_{1}^{(n)} \leq 1$ and $p_{2}^{(n)}=1-p_{1}^{(n)}$. They might have a general dependence on the partial widths $\varepsilon_{i}^{(n)}, i=1,2: p_{i}^{(n)}=p_{i}^{(n)}\left(\varepsilon_{1}^{(n)}, \varepsilon_{2}^{(n)}\right)$. Due to dimensional reasons they can only depend on the ratio $z^{(n)} \equiv \varepsilon_{1}^{(n)} / \varepsilon_{2}^{(n)}$. Thus we write

$$
p_{1}^{(n)}=g\left(z^{(n)}, \omega\right), \quad p_{2}^{(n)}=1-g\left(z^{(n)}, \omega\right) .
$$

Here $\omega$ is a parameter of the distributions, and incorporates the dependence on the replication rates. We also made the plausible assumption, that $g$ has no explicit $n$ (or time) dependence. A general property of $g$ is that it vanishes in the origin $g(0)=0$ since this expresses the obvious fact that if species $B_{1}$ is missing, then the probability to find it in the filaments is zero. Similarly for infinitely large values of $z$ it must be unity: $g(\infty)=1$ which corresponds to the absence of $B_{2}$. Also, due to the fact that $g(z)$ is a probability distribution, we must have $0 \leq g(z) \leq 1$ for all $z \geq 0$. Furthermore, the functional form must be symmetric by interchanging the role of the species. This implies that one must have $g^{\prime}(0) \geq 0$, where the prime denotes derivation with respect to the argument. This implies

$$
p_{2}^{(n)}=g\left(1 / z^{(n)}, 1 / \omega\right)
$$

where the appearance of $1 / \omega$ means that an interchange of the species index brings the parameter in its reciprocal value, as e.g. in the case when $\omega=\sigma_{1} / \sigma_{2}$ (the dependence on the ratio of the replication distances follows from dimensional reasons). The normalization of the probability 
implies

$$
g(z, \omega)+g(1 / z, 1 / \omega)=1
$$

This is a functional equation for $g$. With the above properties and boundary conditions we find that a family of solutions is given by the form:

$$
g(z)=\frac{z^{\alpha}}{z^{\alpha}+\omega}
$$

with $\alpha$ and $\omega$ as two positive parameters. In the range of $0<\alpha<1$ the smaller population is less probable on the boundary but yet with a weight which is weaker than linear in the widths. For $\alpha=0$ there is no widthdependence at all, the probabilities $p_{i}$ are constant. The case $\alpha=1$ and $\omega=1$ corresponds to a homogeneous mixing within the stripe of width $\varepsilon$. For $\alpha>1$ a superdominance is described. In the next section we show that the form (19) of $g(z)$ is indeed in good agreement with numerical simulations, and determine values for parameters $\alpha$ and $\omega$.

The broadening of the average widths is then $c \sigma_{1} p_{1}^{(n)}$ and $c \sigma_{2} p_{2}^{(n)}$ due to species $B_{1}$ and $B_{2}$, respectively. Here the geometrical factor $c$ and parameter $\sigma_{i}=\gamma_{i}-\mu_{i}$ have the same meanings as in the single species problem defined previously.

Thus, similar to (里) the partial width of $B_{i}$ after the $(n+1)$ st step is

$$
\varepsilon_{i}^{(n+1)}=\left[\varepsilon_{i}^{(n)}+c \sigma_{i} p_{i}^{(n)}\right] e^{-\lambda \tau}
$$

for $i=1,2$. Note that in our theory, $c p_{1}$ and $c p_{2}$ can also be interpreted as renormalized geometric factors for each species, due to the screening effects at the boundaries of the stripes. As a consequence, the total width of the stripes changes at a replication as

$$
\varepsilon^{(n+1)}=\left[\varepsilon^{(n)}+c\left(\sigma_{1} p_{1}^{(n)}+\sigma_{2} p_{2}^{(n)}\right)\right] e^{-\lambda \tau} .
$$

For simplicity, the explicit width-dependence (16) of the probabilities has not been written out. For $\sigma_{1}=\sigma_{2}$ we recover the width dynamics of the single species problem, see (田).

Next we turn to the dynamics of the number of individuals. On scales larger than or equal to $\varepsilon^{(n)}$, the total number of individuals $N=N_{1}+N_{2}$ occupied by stripes appears to be a fractal of the same dimension $D_{0}$ as the outflow curve. For simplicity of writing we assume that both species have the same size $\varepsilon_{0}$ (an extension for different sizes is straightforward).

Since the relation between the $\varepsilon^{(n)}$ and the number of individuals $N^{(n)}$ is the same as in the single species model, we can use (8). Thus (21) implies a recursion for the area right before replication as

$$
N^{(n+1)}=e^{-\kappa \tau}\left\{\left[N^{(n)}\right]^{1 /\left(2-D_{0}\right)}+q\left[\sigma_{1} p_{1}^{(n)}+\sigma_{2} p_{2}^{(n)}\right]\right\}_{(22)}^{2-D_{0}}
$$

with $q$ given by (11). Next, we derive the dynamics of the number of individuals $N_{i}^{(n)}$ for species $i$ contained in the stripes. The number of individuals of species $i$ is the portion of the total number $N^{(n)}$ proportional to the partial widths:

$$
N_{i}^{(n)}=N^{(n)} \frac{\varepsilon_{i}^{(n)}}{\varepsilon^{(n)}} .
$$

This is due to the fact that there is no fractal scaling below $\varepsilon^{(n)}$. Since $\varepsilon^{(n)}=\left[\epsilon_{0}^{2} N^{(n)}\right]^{1 /\left(2-D_{0}\right)}$, Eq. (23) leads to

$$
\varepsilon_{i}^{(n)}=N_{i}^{(n)} \epsilon_{0}^{2 /\left(2-D_{0}\right)}\left[N^{(n)}\right]^{\beta} .
$$

As another consequence of 23), the ratio of the partial widths is the ratio of the population numbers:

$$
z^{(n)} \equiv \frac{\varepsilon_{1}^{(n)}}{\varepsilon_{2}^{(n)}}=\frac{N_{1}^{(n)}}{N_{2}^{(n)}}
$$

From Eqs. (20) and 24) we therefore obtain the dynamics of the population numbers as

$$
\begin{aligned}
N_{i}^{(n+1)}\left[N^{(n+1)}\right]^{\beta} & =e^{-\lambda \tau}\left\{N_{i}^{(n)}\left[N^{(n)}\right]^{\beta}\right. \\
& \left.+q \sigma_{i} p_{i}^{(n)}\left(N_{1}^{(n)} / N_{2}^{(n)}\right)\right\}
\end{aligned}
$$

for $i=1,2$. Here exponent $\beta$ is the same expression (12) as in the case of the single species problem, and $q$ is given by (11).

We observe that by dividing the rearranged (26) for $i=1$ by that with $i=2$, one obtains

$$
\frac{\sigma_{1} p_{1}^{(n)}}{\sigma_{2} p_{2}^{(n)}}=M^{(n)}
$$

where

$$
M^{(n)}=\frac{e^{\lambda} N_{1}^{(n+1)}\left[N^{(n+1)}\right]^{\beta}-N_{1}^{(n)}\left[N^{(n)}\right]^{\beta}}{e^{\lambda} N_{2}^{(n+1)}\left[N^{(n+1)}\right]^{\beta}-N_{2}^{(n)}\left[N^{(n)}\right]^{\beta}} .
$$

From this, $p_{1}=1-p_{2}$ is easily found as

$$
p_{1}^{(n)}=\frac{M^{(n)}}{M^{(n)}+\frac{\sigma_{1}}{\sigma_{2}}},
$$

This relation provides us with a method for measuring how the probability $p_{1}(z) \equiv g(z)$ depends on the ratio $z \equiv N_{1} / N_{2}$ at any instant of time. We shall use this observation to extract the form of the $g$ function from numerical results. The ratio of fixed points, $z^{*} \equiv \frac{N_{1}^{*}}{N_{2}^{*}}$ can be calculated from (29) assuming that $N_{2}^{*} \neq 0$ as

$$
g\left(z^{*}\right)=\frac{M^{*}}{M^{*}+\frac{\sigma_{1}}{\sigma_{2}}}
$$


The time continuous limit is obtained by letting both the time lag and the effective replication ranges go to zero so that their ratios remain finite. Thus we define replication velocities

$$
v_{i}=\lim _{\tau \rightarrow 0} \frac{\sigma_{i}}{\tau}
$$

with $i=1,2$ for species $B_{1}, B_{2}$, respectively. In the continuous time limit, the differential equations obtained for the partial widths from (20) read as

$$
\frac{d \varepsilon_{i}}{d t}=-\lambda \varepsilon_{i}+c v_{i} p_{i}\left(\varepsilon_{1} / \varepsilon_{2}\right)
$$

where $p_{1}=g, p_{2}=1-g$.

The differential equation for the number of all individuals follows from (22) as

$$
\frac{d N}{d t}=-\kappa N+q\left(2-D_{0}\right) v N^{-\beta} .
$$

Here

$$
v \equiv p_{1} v_{1}+p_{2} v_{2}
$$

is an average velocity, but note that it is not a constant since the $p_{i}$ depend on the population numbers, and $q$ is given by (11).

The differential equation for the number $N_{i}$ of individuals of the two species can be derived from (32) and the continuum version of (24), i.e., $\varepsilon_{i}=\epsilon_{0}^{2 /\left(2-D_{0}\right)} N_{i} N^{\beta}$. The result is

$$
\begin{array}{r}
\frac{d N_{i}}{d t}=-\kappa N_{i}-q\left(D_{0}-1\right) v N^{-\beta-1} N_{i}+ \\
q v_{i} p_{i}\left(N_{1} / N_{2}\right) N^{-\beta},
\end{array}
$$

with $N=N_{1}+N_{2}$. Here (3) and (12) have been used. By summing over $i$ in (35) one recovers Eq. (33).

An equivalent form is obtained after rearranging terms and taking into account the definition of the average replication velocity (34). It reads

$$
\begin{array}{r}
\frac{d N_{1}}{d t}=-\kappa N_{1}+q N^{-\beta-1}\left[\left(2-D_{0}\right) v N_{1}+\right. \\
\left.\left(v_{1} p_{1} N_{2}-v_{2} p_{2} N_{1}\right)\right],
\end{array}
$$

and an analogous expression for the second species obtained from (36) by interchanging the indices 1 and 2 . It can be clearly seen that the first term of the bracket corresponds to the growth of the total population, while the second describes the effect due to a weighted difference in the population numbers. Expression (35) or (36) represents a strongly coupled set of nonlinear equations with a novel type of power-law behavior (with negative exponent $-\beta$ ). This set of equations is the central result of our paper since it can be considered as a population dynamics describing the coupling of two populations mixing on a fractal, and as we show below, opens up the possibility to have a nontrivial coexistence.
If one of the species, say $B_{2}$, is not present, then $p_{1}=1$, $N_{2}=p_{2}=v_{2}=0$ and hence $v=v_{1}=v_{r}, N=N_{1}$ and Eq. (33) becomes equivalent to (10). The same happens if both species are equivalent, i.e., for $v_{1}=v_{2}$ when $v=v_{r}$.

A simple further equivalent form can be derived by using relative densities $c_{i} \equiv N_{i} / N$. The equations describing the populations then become (by using (36) and (33):

$$
\frac{d c_{1}}{d t}=q N^{-\beta-1}\left(v_{1} p_{1} c_{2}-v_{2} p_{2} c_{1}\right)
$$

with $c_{1}+c_{2}=1$. The temporal change of the densities is determined by the weighted relative difference in the densities. Note that they are multiplicatively coupled to $N^{-\beta-1}$ which is proportional to the average width of the filament covering. For $D_{0}=1$ this is just $1 / N$ and it is the spatial concentration or the density of the total population. For $2>D_{0}>1$ (fractals), this factor is the fractal spatial density of the population as a whole.

\section{COEXISTENCE ANALYSIS: FIXED POINTS AND THEIR STABILITIES}

It is simple and instructive to study the timecontinuous dynamics of the widths $\varepsilon_{i}, i=1,2$ in and around steady states. Assuming, as before, $c=$ const., we find from (32)

$$
\lambda \varepsilon_{i}^{*}=c v_{i} p_{i}\left(\varepsilon_{1}^{*} / \varepsilon_{2}^{*}\right) .
$$

The weighted sum of the fixed points widths (38) gives

$$
\varepsilon_{1}^{*} v_{2}+\varepsilon_{2}^{*} v_{1}=\frac{c v_{1} v_{2}}{\lambda}
$$

From here on, for the stability analysis, we shall use the explicit form (19) for the function $g$. Thus (38) translates into:

$$
\begin{aligned}
& \lambda \varepsilon_{1}^{*}=c v_{1} \frac{\varepsilon_{1}^{* \alpha}}{\varepsilon_{1}^{* \alpha}+\omega \varepsilon_{2}^{* \alpha}} \\
& \lambda \varepsilon_{2}^{*}=c v_{2} \frac{\omega \varepsilon_{2}^{* \alpha}}{\varepsilon_{1}^{* \alpha}+\omega \varepsilon_{2}^{* \alpha}}
\end{aligned}
$$

Formula (39) shows that one species always survives. Without loss of generality, we can choose this to be species $B_{2}$, and everything remains valid with the indices 1 and 2 switched. It is worth defining:

$$
z^{*} \equiv \varepsilon_{1}^{*} / \varepsilon_{2}^{*}
$$

Since $\varepsilon_{2}^{*}$ is not zero, the ratio of the fixed point equations (40,41) yields:

$$
z^{* 1-\alpha}=\frac{v_{1}}{v_{2} \omega} \quad \text { or } \quad z^{*}=0
$$


The first equality describes the $z^{*} \neq 0$ coexistence fixedpoint while the second describes the non-coexistence fixedpoint.

The equations (32) of the continuous case written out explicitely are as follows:

$$
\begin{aligned}
& \frac{d \varepsilon_{1}}{d t}=-\lambda \varepsilon_{1}+c v_{1} \frac{\varepsilon_{1}^{\alpha}}{\varepsilon_{1}^{\alpha}+\omega \varepsilon_{2}^{\alpha}} \\
& \frac{d \varepsilon_{2}}{d t}=-\lambda \varepsilon_{2}+c v_{2} \frac{\omega \varepsilon_{2}^{\alpha}}{\varepsilon_{1}^{\alpha}+\omega \varepsilon_{2}^{\alpha}}
\end{aligned}
$$

The linear stability of a fixed point $\left(\varepsilon_{1}^{*}>0, \varepsilon_{2}^{*}>0\right)$ will be given by the eigenvalues of the stability matrix E, calculated from (44, 45 ) as follows (here we also used (40,41):

$$
\mathbf{E}=\lambda\left(\begin{array}{cc}
-1+\frac{\alpha \lambda}{c v_{2}} \varepsilon_{2}^{*} & -\frac{\alpha \lambda}{c v_{2}} \varepsilon_{1}^{*} \\
-\frac{\alpha \lambda}{c v_{1}} \varepsilon_{2}^{*} & -1+\frac{\alpha \lambda}{c v_{1}} \varepsilon_{1}^{*}
\end{array}\right)
$$

The eigenvalues of $\mathbf{E}$ are easily calculated:

$$
\Lambda_{+}=-\lambda(1-\alpha), \quad \Lambda_{-}=-\lambda
$$

One eigenvalue of the width dynamics is always the negative of the chaotic advection's positive Lyapunov exponent. As long as the parameter $\alpha$ is less than unity, the other eigenvalue is also negative.

We find that for $0<\alpha<1$ coexistence is stable, for $\alpha>1$ it becomes unstable.

The case $\alpha=1$ is special. It follows from (43) that for $\alpha=1$, and $\omega \neq v_{1} / v_{2}$, the non-coexsitence point is the only fixed point, and it is stable. (If $\omega>v_{1} / v_{2}$ then $\left(\varepsilon_{1}^{*}>0, \varepsilon_{2}^{*}=0\right)$ is stable, if $\omega<v_{1} / v_{2}$ then $\left(\varepsilon_{1}^{*}=0, \varepsilon_{2}^{*}>0\right)$ is stable fixed point.) Having $\alpha=1$ with $\omega=v_{1} / v_{2}$ implies that all points fulfilling (39) are fixed points of marginal stability. Thus, stable coexistence is found in the

$$
0<\alpha<1
$$

regime.

Interestingly, the stability conditions are the same for discrete-time dynamics, as well.

In the next section we analyze coexistence in a simple chaotic dynamical system, the Baker map, where we show that $g(z)$ is given by Eq. (19) in this process, and determine the parameters $\alpha$ and $\omega$ from numerical experiments.

\section{NUMERICAL RESULTS}

In this section we present numerical verification of the new type of population dynamical equation we introduced before. We have already shown that coexistence in open flows is possible Scheuring et al., 2000; Károlyi et al., 2000), so we deal only with the quantitative verification of the theoretical results.

For computational simplicity, we use the so-called baker map to model the flow (Toroczkai et al., 2001). This can be considered as a simplified model of stretching and folding in a chaotic flow observed periodically after specified time-intervals. Thus, in this case $\tau$, the time lag between instantaneos multiplications of the species, is an integer number denoting the number of snapshots taken of the flow between two consequtive multiplications. The baker map, acting on the unit square, gives the new location $\left(x^{\prime}, y^{\prime}\right)$ of an individual starting at point $(x, y)$ :

$$
\begin{array}{ll}
x^{\prime}=a x+(1-a) \theta(y-1 / 2), & x \in[0,1], \\
y^{\prime}=\frac{1}{a} y-\left(\frac{1}{a}-1\right) \theta(y-1 / 2), & y \in[0,1],
\end{array}
$$

where $a<1 / 2$ is the parameter of the baker map, and $\theta(x)$ is the Heaviside step function. The action of the baker map is shown schematically in Fig. 3. The area preserving property of this baker map models the incompressibility of realistic hydrodynamical flows, while outflow is modelled by neglecting the area hanging over the edge of the unit square.
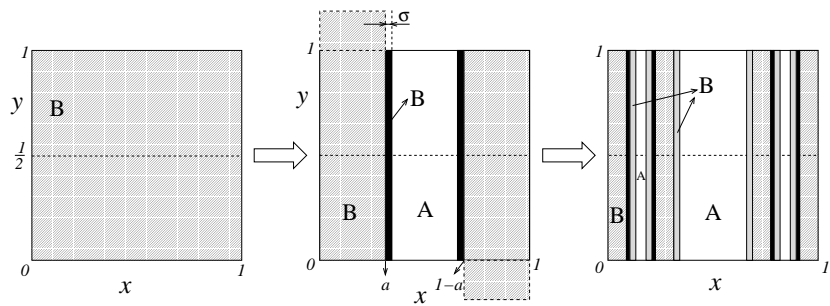

FIG. 3. Two consecutive steps of the baker map and two replications $(\tau=1)$ for the single species model. The bands of width $\sigma$ become occupied by $B$ in each replication. The material hanging over the unit square is discarded.

Starting with any initial conditions, after a few steps of iterations both species will be distributed along narrow filaments parallel to the $y$ axis.

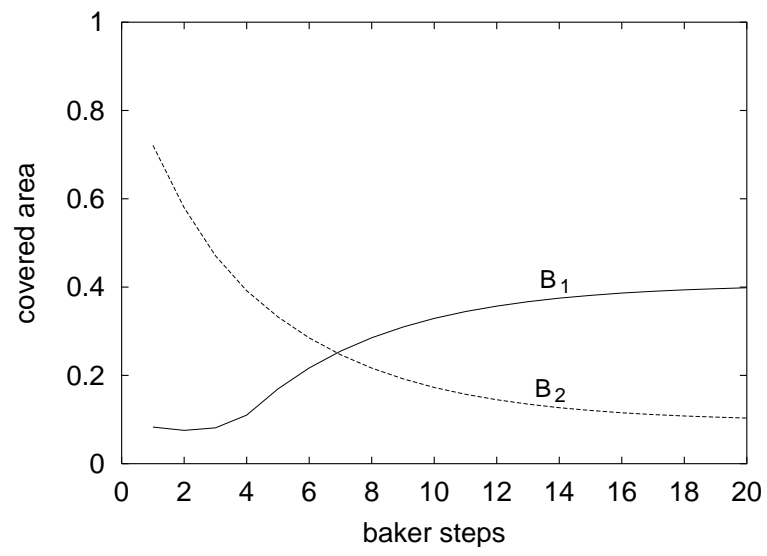


FIG. 4. Reaching the equilibrium states with the coexistence of two species is shown. Initially, two patches of species were placed, one patch of $B_{1}$ in $x \in[0 ; 0.1], y \in[0 ; 1]$, and another patch of $B_{2}$ at $x \in[0.1 ; 1], y \in[0 ; 1]$. The parameter values are $a=0.4$ for the baker map, and $\sigma_{1}=0.003$ $\sigma_{2}=0.001$ for the competing species. The areas covered by the species is shown right after the multiplications taking place. After an initial transient (time-steps 1-4), we have a rapid convergence to the fixed point (time-steps 5-18), after that we have an equilibrium setting in (time-steps 18-20).

After $\tau$ baker steps, individuals of species $B_{i}$ multiply and give birth into a vertical stripe of width $\sigma_{i}$ covered by resource $A$, lying along the borderline of the previously occupied region of species $B_{i}$ parallel to the $y$ axis. In the numerical experiments, we used $\tau=1$, that is, the species multiplied after each baker-step. Regions which are invaded by both species after instantaneous multiplication are divided between them in a ratio of $\sigma_{1} / \sigma_{2}$. It is expected that (26) describes the time-evolution of the species, reaching the fixed-point (30) eventually. Figure shows in a typical case how the equilibrium state with coexistence is reached after about 18 baker steps. Similar results were obtained with various other parameter settings, in accordance with the theoretical results. We checked the validity of the form (30) against the numerical results in steady states. $N_{i}^{*}$ is the fixed-point number of individuals of species $B_{i}$. The fixed point values are found to fulfill (30), see Fig. 5 .

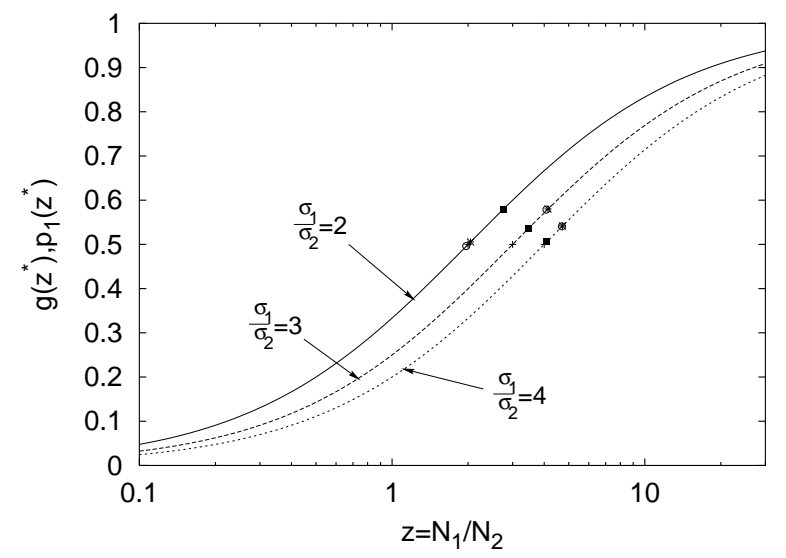

FIG. 5. The dependence of the probabilities $p_{1}$ on $N_{1} / N_{2}$ in the nontrivial fixed points. The initial positions do not influence the fixed point reached. The curve $g(z)=z /\left(z+\sigma_{1} / \sigma_{2}\right)$ is shown with solid line for $\sigma_{1}=0.002, \sigma_{2}=0.001$, with dashed line for $\sigma_{1}=0.003, \sigma_{2}=0.001$, and with dotted line for $\sigma_{1}=0.004, \sigma_{2}=0.001$. All the measured fixed point values fulfill $g\left(z^{*}\right)=z^{*} /\left(z^{*}+\sigma_{1} / \sigma_{2}\right)$. The fixed points are marked by crosses $(a=0.25)$, black squares $(a=0.3)$, starts $(a=0.35)$, and circles $(a=0.4$ as the baker parameter $)$.

Next we check the validity of (26) for the timeevolution before reaching the convergence. We measure the population numbers in discrete time $n$ and use relation (29) to extract the form of the probability distri- bution $g$. Figure 6 shows $p_{1}$ as a function of $N_{1} / N_{2}$ for fixed parameter values, but for various initial conditions. There is a single function covering the measured points which can well be fitted by the form $g_{1}(z)=z^{\alpha} /\left(z^{\alpha}+\omega\right.$. In all cases $\alpha<1$ was measured indicating that the coexistence fixed point is stable. Also note that $\omega \approx\left(\sigma_{1} / \sigma_{2}\right)^{\alpha}$ was found in all experiments, which implies that (18) holds.

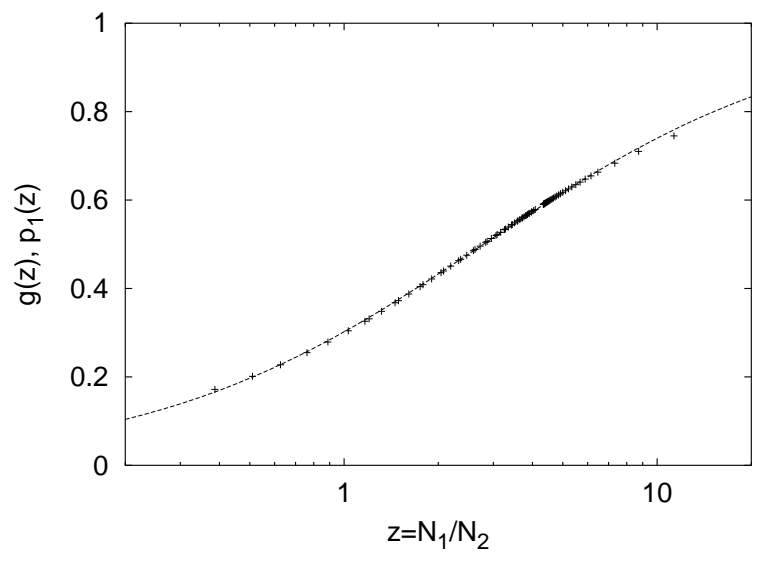

FIG. 6. The dependence of the probabilities $p_{1}$ on $N_{1} / N_{2}$ with $\sigma_{1}=0.003, \sigma_{2}=0.001$ values, and with five different initial conditions. The parameter of the baker map was $a=0.4$. The initial positions do not influence the fixed point reached. Solid line shows the function $g(z)=z^{\alpha} /\left(z^{\alpha}+\omega\right)$ with $\alpha=0.818 \pm 0.002$ and $\omega=2.312 \pm 0.006$. The parameter of the baker map was $a=0.4$.

We also measured how $\alpha$ depends on the parameter of the baker map, or, on the fractal dimension $D_{0}$ of the outflow curve of the chaotic saddle. We found that $\alpha=0.79 \ln a+1.54$, see Fig. \&. Using the fact that $D_{0}=\ln 2 / \ln (1 / a)$, we obtain $\alpha=1.54-0.55 / D_{0}$.

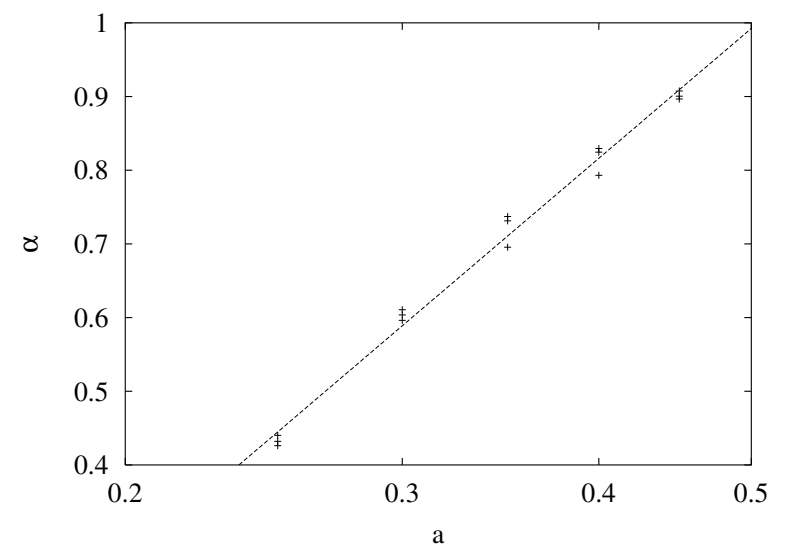

FIG. 7. Dependence of $\alpha$ on the parameter $a$ of the baker map. Various points are measured values for multiple $\sigma_{1} / \sigma_{2}$ ratios. The dashed line is the function $\alpha(a)=0.79 \ln a+1.54$. 


\section{DISCUSSION}

We derived a novel type of coupled population dynamic equations for two populations competing on a fractal set provided by open chaotic flows. The equation for the number of species in a given fixed range of the flow can be written in the general scheme (cf. 36)

$$
\begin{aligned}
& \frac{d N_{1}}{d t}=\alpha_{1}\left(\frac{N_{1}}{N}\right) N_{1}^{-\beta}-\kappa N_{1}, \\
& \frac{d N_{2}}{d t}=\alpha_{2}\left(\frac{N_{2}}{N}\right) N_{2}^{-\beta}-\kappa N_{2} .
\end{aligned}
$$

The coefficients $\alpha_{i}$ of the replication terms depend on the relative densitites (denoted by $N_{i} / N \equiv c_{i}$ ) only. Their explicit form follows from (36). For example,

$$
\alpha_{1}\left(\frac{N_{1}}{N}\right)=q\left(\frac{N_{1}}{N}\right)^{\beta}\left[\left(1-D_{0}\right) v \frac{N_{1}}{N}-v_{1} p_{1}\right] .
$$

The structure of these equations is similar to that of (2), (2) or $(13,14)$. The time derivative is the sum of a gain term and a loss term, but now the gain term contains a nontrivial negative power of the population number and is coupled to the other population in a nonlinear way. These equations describe the population dynamics in an imperfectly mixed enviroment of dimension $1<D_{0}<2$. The fractality $D_{0}$ of the mixing region (in our case of the outflow curve) appears in the power $\beta=\left(2-D_{0}\right) /\left(D_{0}-1\right)$. In this set of equations a phenomenological function $\left(p_{1}\right)$ is also present characterizing the probability that a given population is on the surface of the fractal support with free access to the single available resource. Based on general arguments and a simple model, this function turned out to be a normalized power law distribution of the type of (19). This form expresses a kind of "advantage of rarity" principle: for exponent $0<\alpha<1$ the derivative is infinite in the origin, a very small increase in the size of the weaker population leads to a drastical increase of the probability for being on the free surface and hence to grow. On the contrary, for $\alpha>1$, only a relatively large population size has considerable growing probability, in this case the weaker population dies out. It is worth mentioning that in the gedankenexperiment of Section "Coexistence of competing species", we did not see this effect since we had an initial stage at which the weaker population does not yet influence the stronger one. The probability of being on the surface was assumed to be constant. It is the interaction between the two populations which leads to the power law distribution. Its exponent is deternined by the flow and the biological process. With exponents larger than unity this form does not allow for coexistence. The presented mathematical forms and the conditions for coexistence remain valid if $m>2$ species live in open chaotic flow, a numerical evidence for which has been reported by Károlyi et al. (2000). It is natural to expect that the probabilities $p_{i}, i=1, \ldots, m$ appear in the generalized form of $p_{i}=\left(\omega_{i} \varepsilon_{i}^{\alpha}\right) /\left(\sum_{i=1}^{m} \omega_{i} \varepsilon_{i}^{\alpha}\right)$, where $\varepsilon_{i}$ are the partial width of the species and $\omega_{i}$ are phenomenological constants.

Although in the numerical simulation, based on the baker map as a model flow, we only found stable coexistence, we also carried out simulations where the biological process was not based on parallel stripes filled out homogeneously with individuals, as assumed in our theory. In these cellular automaton-like simulations the replication and competition process is carried out on a uniform rectangular grid of lattice size $\epsilon_{0}$. This $\epsilon_{0}$ can be considered as the smallest distance between the individuals, or the linear size of a single individual below which there is hard-core exclusion among them. Individuals of each species can occupy the center of each grid-cell. When they are advected by the flow into another gridcell during the time $\tau$, they are instantaneously placed to the center of that grid-cell. During reproduction, they give birth to new individuals in the surrounding empty grid-cells, whose centers are within a distance $\sigma_{i}$. If more than one species tries to give birth into the same gridcell, only one of them will be able to do so according to one of the following rules: rule $I$ : both species can win this competition in each cell with equal probability, or rule II: both species can win this competition in each cell with probability proportional to the number of individuals of the same species intending to give birth there, or rule III: always the better competitor (with higher $\sigma$ ) wins. Our results show that the coexistence depends on which rule has been applied. In some cases one of the populations was competed out, but even in such cases the distribution function was found to be of the shown form, with an exponent $\alpha>1$, in full harmony with the theory. It is worth mentioning that with the same rules on the lattice, in previous simulations (Károlyi et $a l ., 2000$ ) for the more realistic fluid dynamical case of a flow around an obstacle we always found coexistence. This indicates that the boundary layer present around the obstacle enhances the chances of survival.

Our theory does not describe the effects of diffusion. Besides the fact that for individuals of small but macroscopic mass and size, like eg. phytoplankton, diffusion is not believed to be important, it can be shown Tél et al., 2000) that weak diffusion in such models only renormalizes the replication rates. As a consequence, the cut-off scale below which fractality cannot be observed is somewhat increased, but the population dynamical equations remain unchanged.

In this theory the location dependence of the death and replication rates is not taken into account. Such effects can be studied in numerical simulations (Károlyi et al., 2000: Santoboni et al., 2001) and are not expected to change the essence of our findings.

In conclusion, we have shown that a particle-like (microscopic) model of individuals competing for a single resource around a fractal outflow curve of a chaotic flow 
leads, on the level of the total number of individuals, to dynamical equations with unusual singular terms. These describe enhanced competition due to inhomogeneous mixing and a kind of advantage of rarity property. The appearance of unusual population dynamical equations can be expected in general in all cases where the individual dynamics is not taking place on full compact regions of the space but are restricted to fractal subsets of it.

\section{ACKNOWLEDGEMENTS}

Z.T. was supported by the Department of Energy under contract W-7405-ENG-36. Support from the Hungarian Science Foundation (OTKA T032423, F029637) the US-Hungarian Joint Fund (Project No. 501) and the MTA/OTKA/NSF Fund (Project No. Int. 526) is acknowledged. G.K. and I. Sch were supported by the Bolyai grant. The authors gratefully acknowledge discussions with C. Grebogi, E. Ben-Naim, T. Czárán and E. Szathmáry.

Aref, H. (Ed.) 1994: Special issue on Chaotic Advection. Chaos, Solitons, Fractals 4, No. 6.

Arístegui, J.; Tett, P.; Hernández-Guerra, A.; Basterretxea, G.; Montero, M.F.; Wild, K.; Sangrá, P.; Hernández-León, S.; Cantón, M.; García-Braun, J.A.; Pacheco, M. \& Barton, E.D. 1997: The influence of island-generated eddies on chlorophyll distribution: a study of mesoscale variation around Gran Canaria. Deep-See Research 44, 71-96.

Bartha, S.; Czárán, T. \& Scheuring, I. 1997: Spatiotemporal scales of non-equilibrium community dynamics: a methodological challenge. N. Z. J. of Ecol. 21, 199-206.

Boerlijst, M.C. \& Hogeweg, P. 1991: Physica D 48, 17-28.

Chesson, P. 2000: Mechanism of maintenance of species diversity. Annu. Rev. Ecol. Syst. 31, 343-366.

Connell, J.H. 1978: Diversity in tropical rain forests and coral reefs. Science 199, 1302-1310.

Czárán, T. \& Szathmáry, E. 2000: In: Dieckmann, U.; Law, R. \& Metz, J.A.J. (Eds): The Geometry of Ecological Interactions: Simplifying Spatial Complexity. Cambridge Univ. Press, Cambridge.

Eigen, M. 1971: Naturwissenschaften 58, 465-523.

Eigen, M. \& Schuster, P. 1979: The Hypercycle. Springer, Berlin.

Gaedeke, U. \& Sommer, U. 1986: The influence of the frequency of periodic disturbances on the maintenance of phytoplankton diversity. Oecologia 71, 98-102.

Gause, G.F. \& Witt, A.A. 1935: Behavior of mixed populations and the problem of natural selections. Am. Nat. 69, 596-609.

Gurney, W.S.C. \& Nisbet, R.M. 1998: Ecological Dynamics. Oxford Univ. Press, Oxford.
Hardin, G. 1960: The competitive exclusion principle. Science 131, 1292-1298.

Holm, N.G. 1992: Marine hydrothermal systems and the origin of life. Origins Life Evol. Biosphere 22, 5.

Hsu, G.H.; Ott, E \& Grebogi, C. 1988: Phys. Lett. A 127, 199.

Huston, M.A. 1979: General hypothesis of species diversity. Am. Nat. 113 81-101.

Hutchinson, G.E. 1961: The paradox of the plankton Am. Nat. 95, 137-147.

Jung, C. \& Ziemniak, E. 1992: Hamiltonian scattering chaos in a hydrodynamical system. J. Phys. A 25, 3929-3943.

Kantz, H. \& Grassberger, P. 1985: Repellers, semiattractors, and long-lived chaotic transients. Physica D 17, 75 .

Károlyi, G. \& Tél, T. 1997: Chaotic tracer scattering and fractal basin boundaries in a blinking vortex-sink system. Physics Reports 290, 125-147.

Károlyi, G.; Péntek, Á.; Toroczkai, Z.; Tél, T. \& Grebogi, C. 1999: Chemical or biological activity in open chaotic flows. Phys. Rev. E 59 5468-5481.

Károlyi, Gy.; Péntek, Á.; Scheuring, I.; Tél, T. \& Toroczkai, Z. 2000: Open chaotic flow: the physics of species coexistence. Proc. Natl. Acad. Sci. USA 97 13661-13665.

Lamb, H. 1932: Hydrodynamics. Cambridge University Press, Cambridge.

Maynard Smith, J. 1983: Models of evolution. Proc. Roy. Soc. London B 219, 315-325.

Maynard Smith, J. \& Szathmáry, E. 1995: The major transitions in evolution. Freeman, Spektrum, Oxford.

Péntek, Á.; Tél, T. \& Toroczkai, Z. 1996: Transient chaotic mixing in open hydrodynamical flows. Int. J. Chaos and Bifurcations 6, 2619-2625.

Poole, A.; Jeffares, D. \& Penny, D. 1999: Early evolution: prokaryotes, the new kids on the block. BioEssays 21, 880889.

Reynolds, C.S. 1993: Scales of disturbance and their role in plankton ecology. Hydrobiologia 249, 157-171.

Reynolds, C.S. 1998: The state of freshwater ecology. Freshwater Biology 39, 741-753.

Santoboni, G.; Nishikawa, T.; Toroczkai, Z. \& Grebogi, C. 2001: Autocatalytic reactions of active particles with phase distribution. Preprint.

Scheuring, I. 2000: Avoiding Catch-22 of early evolution by stepwise increase in copying fidelity. Selection 1-3, 135-145. Scheuring, I.; Károlyi, G.; Péntek, Á.; Toroczkai, Z. \& Tél, T. 2000: A Model for resolving the plankton paradox: coexistence in open flow. Freshwater Biology 45, 123-133.

Shariff, K.; Pulliam, T.H. \& Ottino, J.M. 1991: A dynamical systems analysis of kinematics in the time-periodic wake of a circular cylinder. Lect. Appl. Math. 28, 613-646.

Sommer, U.; Padisák, J.; Reynolds C.S. \& Juhász-Nagy, P. 1993: Hutchinson's heritage: the diversity disturbance relationship in phytoplankton. Hydrobiologia 249, 1-8.

Sommerer, J.C.; Ku, H.-C. \& Gilreath, H.E. 1996: Experimental evidence for chaotic scattering in a fluid wake. Phys. Rev. Lett. 77, 5055-5058.

Tél, T. 1990: In: Bai-Lin, H. (Ed.): Directions in chaos, Vol. 3., pp. 149-211. World Scientific, Singapore.

Tél, T.; Károlyi, G.; Péntek, Á.; Scheuring, I.; Toroczkai, Z.; 
Grebogi, C. \& Kadtke, J. 2000: Chaotic advection, diffusion, and reactions in open flows. Chaos 10, 89-98.

Tilman, D. \& Pacala, S. 1993: In: Ricklefs, R.E. \& Schluter, D.: Species diversity in ecological communities: The maintenance of species richness in plant communities. pp. 13-25, Univ. of Chicago Press.

Toroczkai, Z.; Károlyi, G.; Péntek, Á.; Tél, T. \& Grebogi, C. 1998: Advection of active particles in open chaotic flows. Phys. Rev. Lett. 80, 500-503.

Toroczkai, Z.; Károlyi, G.; Péntek, Á.; Tél, T.; Grebogi, C. \& Yorke, J.A. 1997: Wada dye boundaries in open hydrody- namical flows. Physica A 239, 235-243.

Toroczkai, Z.; Károlyi, G.; Péntek, Á. \& Tél, T. 2001: Autocatalytic reactions in systems with hyperbolic mixing: Exact results for the active baker map. J. Phys. A, 34, 5215-5235. Wächtershäuser, G. 1994: Life in a ligand sphere. Proc. Natl. Acad. Sci. USA 91, 4283-4287.

Wilson, J.B. 1990: Mechanisms of species coexistence: twelve explanations for Hutchinson's 'paradox of the plankton': evidence from New Zealand plant communities. $N$. $Z$. J. of Ecol. 43, 17-42. 